Research Article

\title{
Experimental Investigation on Strength and Deformation Characteristics of Red Sandstone at Strain Rates of $10^{-2} \sim 55 \mathrm{~s}^{-1}$
}

\author{
Jie Shi, ${ }^{1}$ Zongmu Luo $\mathbb{D},{ }^{2}$ Huachao Liu $\mathbb{D}^{1},{ }^{1}$ Dan Wang, ${ }^{1}$ Haipeng Shen, ${ }^{3}$ and Zeng Li $^{2}$ \\ ${ }^{1}$ National Defense School, Army Engineering University of PLA, Nanjing 210007, China \\ ${ }^{2}$ State Key Laboratory of Disaster Prevention and Mitigation of Explosion and Impact, Army Engineering University of PLA, \\ Nanjing 210007, China \\ ${ }^{3}$ School of Field Engineering, Army Engineering University of PLA, Nanjing 210007, China
}

Correspondence should be addressed to Zongmu Luo; langdon0114@163.com and Huachao Liu; 273750748@qq.com

Received 6 May 2020; Revised 11 June 2020; Accepted 3 August 2020; Published 13 August 2020

Academic Editor: Yanchao Shi

Copyright (C) 2020 Jie Shi et al. This is an open access article distributed under the Creative Commons Attribution License, which permits unrestricted use, distribution, and reproduction in any medium, provided the original work is properly cited.

\begin{abstract}
The mechanical properties of rocks under low to intermediate strain rate are of great importance for seismic engineering, rock impact, and blasting excavation. To study the strength and deformation characteristics of sandstone subjected to low-medium speed impact loading, the complete stress-strain relationships of uniaxial compression at strain rates of $10^{-2} \sim 55 \mathrm{~s}^{-1}$ were obtained utilizing MTS and drop weight impact test devices. It is indicated that the dynamic compressive strength of sandstone in the range of intermediate strain rate increases approximately linearly with the strain rate under the quasi-static loading condition, while increasing nonlinearly under the dynamic loading condition. The deformation and fracture process of sandstone still consists of pore compaction stage, elastic deformation stage, instable microcrack propagation stage, and brittle fracture stage. The peak stress, critical strain, and residual strain increase with an increase in the strain rate, and the corresponding fracture mode changes from shear failure to split failure. The evolution law of total absorbed strain energy with deformation coincides with that of stored elastic strain energy for sandstone at the intermediate strain rate. The effect of the strain rate on elastic strain energy is more significant than that of dissipated strain energy. Furthermore, both the brittleness and fracture degree of sandstone become more remarkable with the strain rate increasing.
\end{abstract}

\section{Introduction}

Dynamic rock mechanics involves the strength and deformation behavior of rock-like materials (e.g., rock and concrete) subjected to dynamic impacts. In general, the mechanical properties and fracture behavior of materials change with an increase in the loading rate. The sources of dynamic loads originate from explosion, shock, and earthquake vibration. In fact, rock engineering often involves the dynamic loading scenarios, such as excavation engineering, civil engineering, blasting engineering, projectile impact, seismic events, and rock collapse. The loading rate is an important index corresponding to different velocities of impacts for rock medium. It is well recognized that different rocks may exhibit diverse mechanical responses with the change of the strain rate [1]. The range of the strain rate can be roughly divided into low strain rate (LSR: $10^{-5} \sim 10^{-2} \mathrm{~s}^{-1}$ ), intermediate strain rate (ISR: $10^{-2} \sim 10^{2} \mathrm{~s}^{-1}$ ), high strain rate (HSR: $10^{2} \sim 10^{4} \mathrm{~s}^{-1}$ ), and ultrahigh strain rate (UHSR: higher than $10^{4} \mathrm{~s}^{-1}$ ). Figure 1 shows a variety of experimental equipment for the application of the above strain rate range. For decades, considerable efforts have been made on the strain rate effect on mechanical properties of various rocks and its underlying physical mechanisms. Huang and Liu [2] carried out low loading rate loading experiments on coal and rock. The results showed that the increase of the strain rate caused more serious damage to the internal structure of coal and rock. Grote et al. [3], Zhang and Zhao [4], and Ma et al. [5, 6] performed low-speed and high-speed impact tests on rocks in the strain rate ranges of $10^{-5} \sim 10^{-2} \mathrm{~s}^{-1}$ and $10^{1} \sim 10^{2} \mathrm{~s}^{-1}$ with the hydraulic testing machine and the large-diameter SHPB test apparatus, 


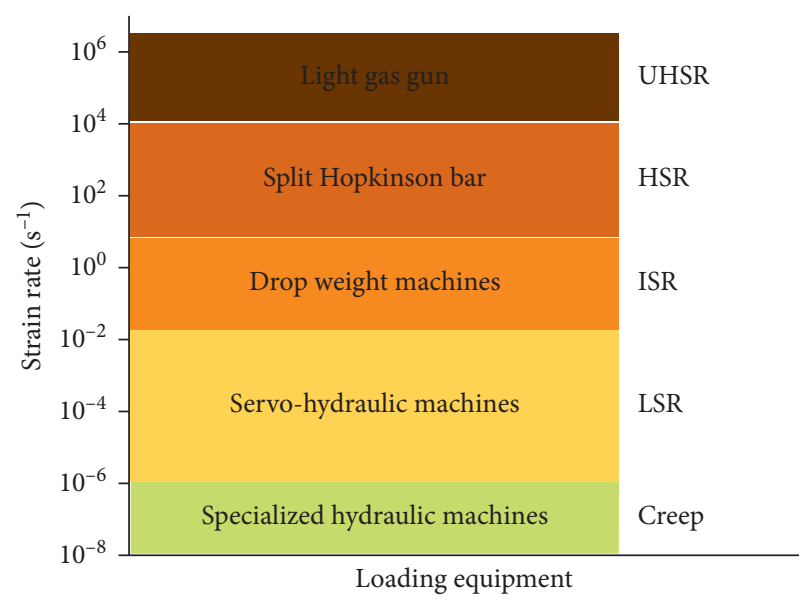

FIGURE 1: Classification of strain rate and loading equipment.

respectively. It is indicated that rock materials present obvious strain rate hardening effect, that is, the peak strength, strain, and elastic modulus of the rock constantly increase with the strain rate increasing. Shang et al. [7] obtained the material state equation of granite by conducting plate impact test on granite at ultrahigh strain rate of $10^{5} \sim 10^{6} \mathrm{~s}^{-1}$.

It is of great significance for the prevention and control of underground engineering disasters such as blasting shock, rock burst, and mine vibration in sandstone medium. It is generally believed that the dynamic strength and cohesion of sandstone are positively correlated with the loading strain rate and the internal friction angle is negatively correlated with the loading strain rate, whereas the effect of the strain rate on elastic modulus is not evident [8-10]. Under the condition of biaxial stress, the dynamic compressive strength of sandstone increases with the increase of strain rate and prestress $\sigma_{2}$, while it decreases with the increase of prestress $\sigma_{1}$ [11]. In the case of triaxial stress, the dynamic compressive strength of sandstone increases linearly with the increase of confining pressure, and the secant modulus also increases with the increase of confining pressure [9]. In addition, Zhou et al. [12] explored the effect of saturated water on dynamic fracture of sandstone. The study showed that free water had a delayed effect on crack propagation speed. Under the same loading rate, the dynamic initiation toughness and expansion toughness of saturated samples were significantly lower than that of dry samples, while the energy absorbed by them was also lower than that of dry samples. Zhang et al. [13] and Luo et al. [14] analyzed the dynamic mechanical characteristics of sandstone under cyclic freezing-thawing conditions. It is indicated that the dynamic compressive strength decreases exponentially with an increase in freezing-thawing times, while it increases linearly with the strain rate increasing. The brittleness of sandstone increases with an increase in freezing-thawing times. Liu and $\mathrm{Xu}$ [15] investigated the effect of the strain rate on dynamic compression mechanical behavior of sandstone at high temperature. The results prove that the enhancement of the strain rate on dynamic compressive strength, critical strain, and energy absorption ratio still exists at high temperature, but the effect on elastic modulus is not obvious. Liu et al. [16] investigated the propagation of shock wave in wet and dry sandstone. The study reveals that the velocity of shock wave in sandstone increases linearly with an increase in impact velocity under the same water content. Free water increases the velocity of the shock wave in the material.

As aforementioned, current research on the mechanical properties of rock-like materials in the high strain rate and the low strain rate regime is relatively systematic, while there are few reports regarding the mechanical behavior of rocks in the range of the intermediate strain rate due to the limitations of experimental devices and technologies. In this paper, uniaxial compression loading experiments within the strain rate range of $10^{-2} \sim 55 \mathrm{~s}^{-1}$ were carried out on sandstone samples using MTS hydraulic test and INSTRON drop weight impact test apparatus. The complete stress-strain relationships of red sandstone at different strain rates are obtained to estimate the effect of the loading strain rate on the peak strength, deformation, failure mode, energy dissipation, and brittleness.

\section{Experimental Procedure}

2.1. Sample Preparation. The tested sandstone were derived from a quarry in Linyi, Shandong province, China. To facilitate transportation, the sandstone was exploited at a depth of $20 \mathrm{~m}$ and cut into plate with the sizes of $1000 \times 400 \times 100 \mathrm{~mm}^{3}$. The cylinder samples with diameters of $50 \mathrm{~mm}$ and $30 \mathrm{~mm}$ were then cored with a rock coring machine. The end face and circumference of the samples were carefully polished with a grinding machine to make the end face parallel error and side vertical error meet the experimental requirements. Finally, the standard cylinder sample [17] with dimensions of $\Phi 50 \mathrm{~mm} \times 100 \mathrm{~mm}$ and $\Phi 30 \mathrm{~mm} \times 60 \mathrm{~mm}$ were prepared for the quasi-static uniaxial compression test and dynamic impact compression test.

2.2. Experimental Equipment and Program. The quasi-static uniaxial compression test was carried out with the MTS647.250 servo-hydraulic material testing device (Figure 2(a)). The maximum axial loading force is $2500 \mathrm{kN}$, and the measurement error of deformation and loading force is less than $1 \%$. The displacement loading mode was adopted and the loading speed was set at $1 \mathrm{~mm} / \mathrm{s}$ and $10 \mathrm{~mm} /$ $s$ in quasi-static compression test, respectively. According to the time-history curves of force and displacement monitored by the testing system, the stress-strain curves of the samples under quasi-static compression can be obtained. The INSTRON (CEAST 9350) drop weight impact apparatus (Figure 2(b)) was used for dynamic compression test. The initial height of the hammer can be set between $0.3 \mathrm{~m}$ and $1.1 \mathrm{~m}$ to achieve an impact speed range between $0.77 \mathrm{~m} / \mathrm{s}$ and $24 \mathrm{~m} / \mathrm{s}$. In the impact loading test, two different hammers of $16.175 \mathrm{~kg}$ and $21.175 \mathrm{~kg}$ were selected with impact velocities of $2.5 \mathrm{~m} / \mathrm{s}, 3 \mathrm{~m} / \mathrm{s}, 3.5 \mathrm{~m} / \mathrm{s}$, and $4 \mathrm{~m} / \mathrm{s}$, respectively. In the center of the sample, the resistance strain gauges connected to the dynamic strain instrument were pasted along the axial and circular direction. The stress-strain curve of the 


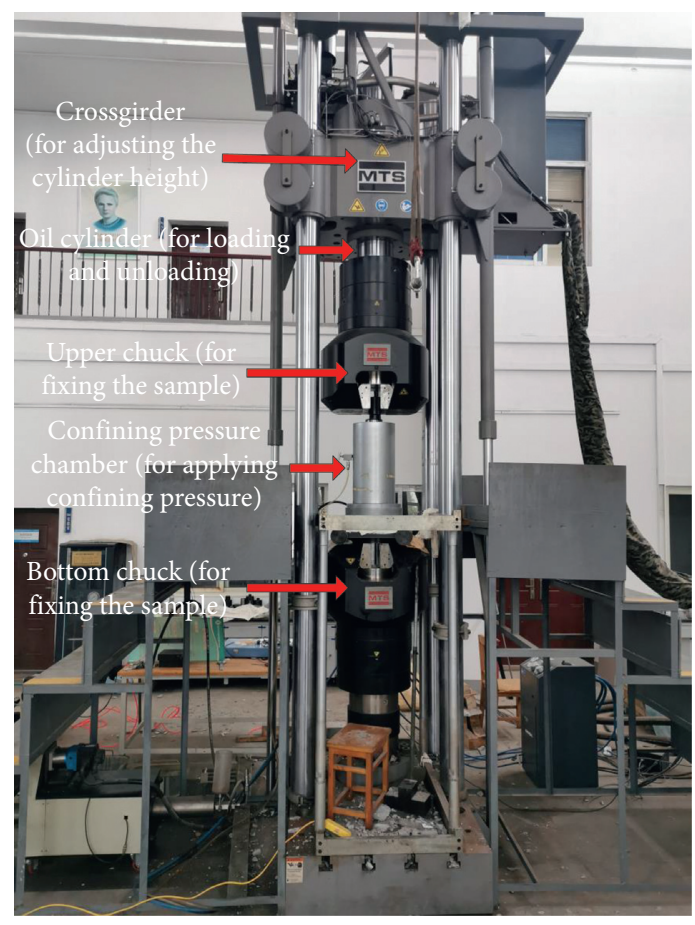

(a)

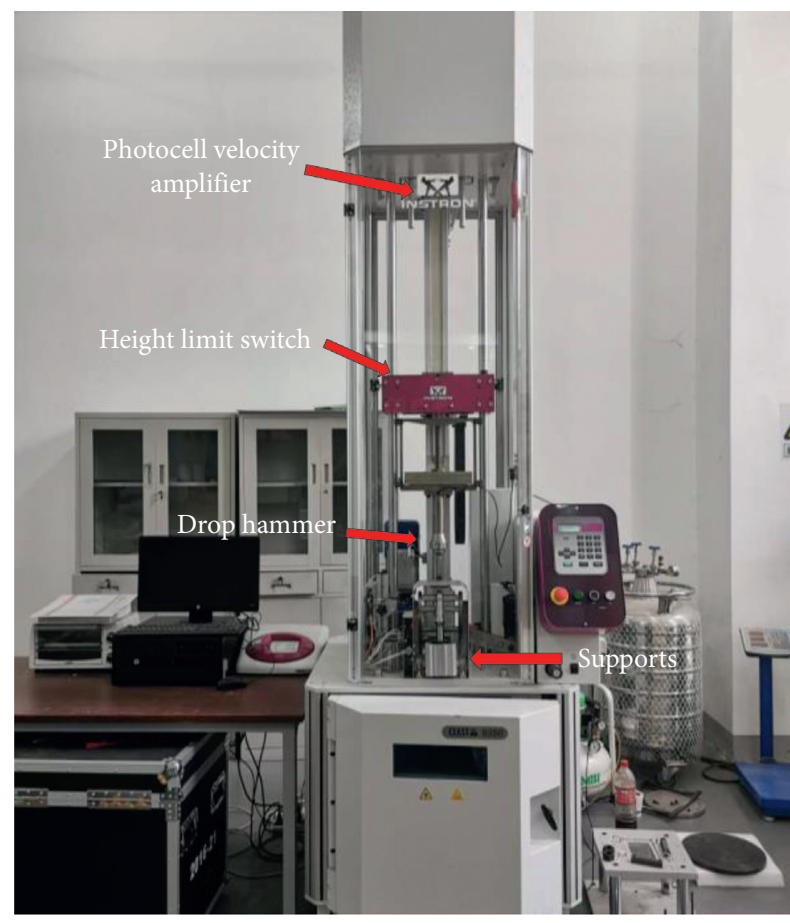

(b)

Figure 2: Experiment apparatus. (a) MTS 647.250 testing machine. (b) Drop weight impact testing machine.

specimen under dynamic compression can be derived by combining the stress time-history curve collected by the force sensor on the hammer and the strain time-history curve collected by the dynamic strain instrument. Meanwhile, a high-speed camera was used to record the macroscopic crack development process of samples under impact loads. Sandstone samples with similar density and porosity were chosen to reduce the discreteness of experimental data.

\section{Results and Discussion}

3.1. Characteristics of Stress-Strain Curve. Figure 3 presents the complete stress-strain relationships of sandstone samples selected for the quasi-static compression test at the strain rate of $10^{-2} \mathrm{~s}^{-1}$ and $10^{-1} \mathrm{~s}^{-1}$. Figure 4 depicts the stressstrain curves of eight sandstone samples selected for the drop weight impact compression test with different hammer weights at different intermediate strain rates.

It can be seen from Figure 3 that the deformation process of sandstone under compression has undergone pore compaction stage, elastic deformation stage, instable fracture propagation stage before failure, and the descending stage after failure. By comparing the curves at two loading strain rates, it can be found that the pore compaction stages basically coincide under quasi-static loading since the samples were prepared with similar initial density, porosity, and other physical properties. Meanwhile, the elastic deformation stage of different samples is also similar, implying the strain rate has no remarkable influence on the elastic modulus of sandstone in the range of

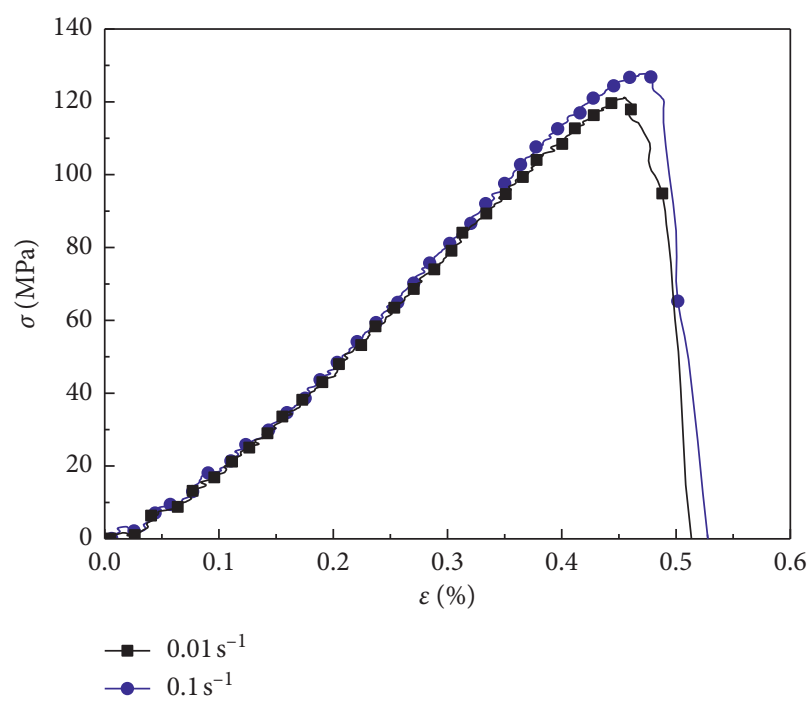

Figure 3: Stress-strain curves at different strain rates under quasistatic compression.

$10^{-2} \sim 10^{-1} \mathrm{~s}^{-1}$. The peak strength of sandstone at strain rate of $10^{-1} \mathrm{~s}^{-1}$ is about 6.5 MPa higher than that of at strain rate of $10^{-2} \mathrm{~s}^{-1}$. The critical strain of sandstone at strain rate of $10^{-1} \mathrm{~s}^{-1}$ is also $1.8 \%$ larger than that of at strain rate of $10^{-2} \mathrm{~s}^{-1}$. As can be seen in Figure 4, the elastic deformation stage under impact load is discrete. The average measured elastic modulus is about $18.13 \mathrm{GPa}$, which is smaller than that under quasi-static compression. This may be because the measured axial strain is 


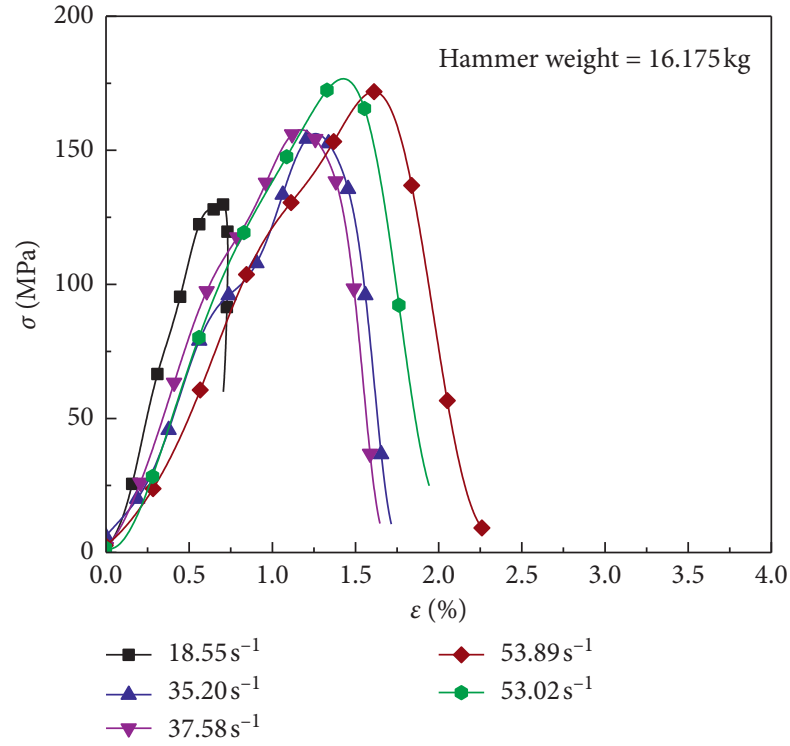

(a)

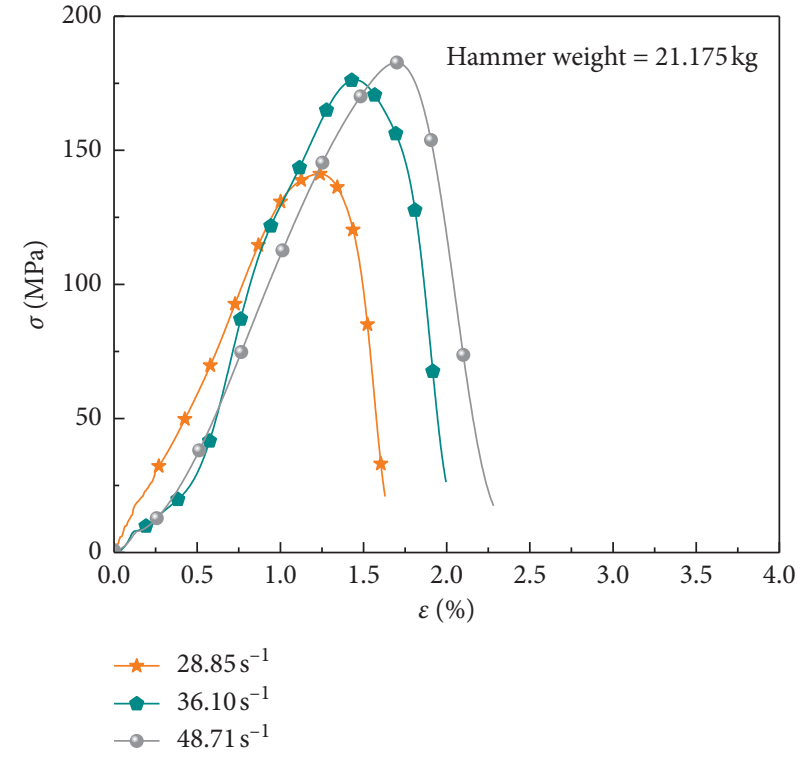

(b)

FIGURE 4: Stress-strain curves at different strain rates under dynamic compression.

larger than the actual one, resulting in a smaller elastic modulus. The steep descent section after failure demonstrates the brittle failure characteristics of sandstone. The peak stress exhibits an obvious rising trend with an increase in the strain rate, which indicates that the compressive strength also has a significant rate effect in intermediate strain rate zone. Compared with Figures 4(a) and 4(b), hammer weight also affects the peak stress of sandstone. Under similar strain rate, the increase of hammer weight leads to an increase in peak stress, which is because the greater the weight of the hammer is, the more energy is transferred to the sample.

3.2. Effect of Strain Rate on Compressive Strength. Figure 5 shows the relation of the compressive strength of sandstone and loading strain rate in the range of $10^{-2} \sim 55 \mathrm{~s}^{-1}$. It can be seen that the compressive strength gradually increases with an increase in the strain rate, indicating that the compressive strength has an obvious strain rate effect, which is consistent with the findings by [18-20]. With the increase of the strain rate, the number of cracks in the failure process increases and the absorbed energy increases, while the crack propagation lags behind the loading speed. According to the impulse theorem, the delayed deformation leads to stress enhancement, which leads to the final improvement of dynamic compressive strength. The underlying physical mechanisms of the strain rate effect of materials are from the perspectives of inertia effect [21], Stefan viscosity effect [22], dynamic fracture effect [23], and thermal activation effect [24].

The ratio of dynamic compressive strength to uniaxial compressive strength at $10^{-2} \mathrm{~s}^{-1}$ strain rate was defined as the dynamic increase factor (DIF). The strength data tested at the intermediate strain rate was plotted in the monologarithmic coordinate, as shown in Figure 6. It is seen that, with the increase of the strain rate, the dependence of

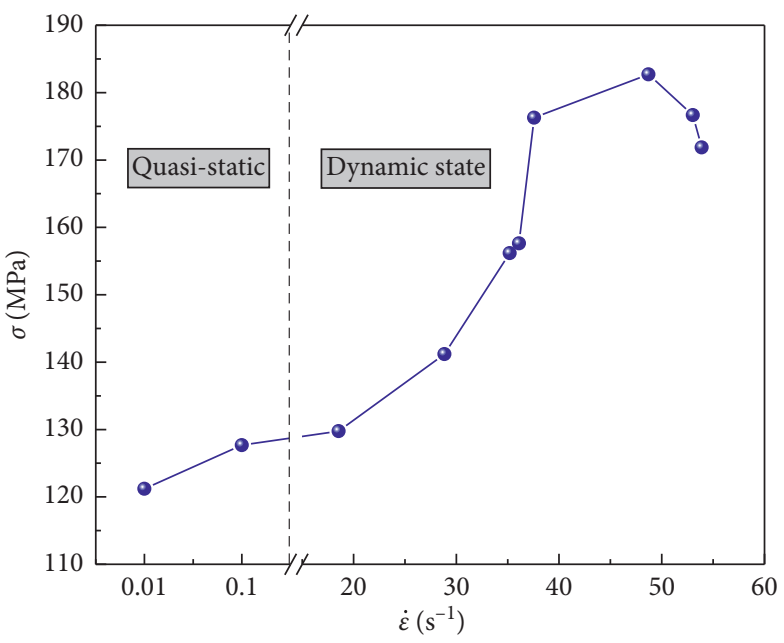

FIGURE 5: Relationship between compressive strength and strain rates.

compressive strength on the strain rate gradually increases. As the strain rate exceeds $10^{1} \mathrm{~s}^{-1}$, the DIF value rapidly increases from 1.08 to 1.47 . Considering that the different rate sensitivity of DIF under quasi-static condition and dynamic condition, the linear and binomial functions are utilized to describe the relationship:

$$
\begin{cases}\text { DIF }=1.06+0.02 \lg \dot{\varepsilon}, & 10^{-2} \mathrm{~s}^{-1} \leq \dot{\varepsilon}<18.55 \mathrm{~s}^{-1}, \\ \text { DIF }=0.74-0.15 \lg \dot{\varepsilon}+0.33(\lg \dot{\varepsilon})^{2}, & 18.55 \mathrm{~s}^{-1} \leq \dot{\varepsilon}<53.89 \mathrm{~s}^{-1} .\end{cases}
$$

3.3. Effect of Strain Rate on Failure Mode. Figure 7 shows the development law of the deformation with the change of the strain rate. Most of the critical strain and residual strain 


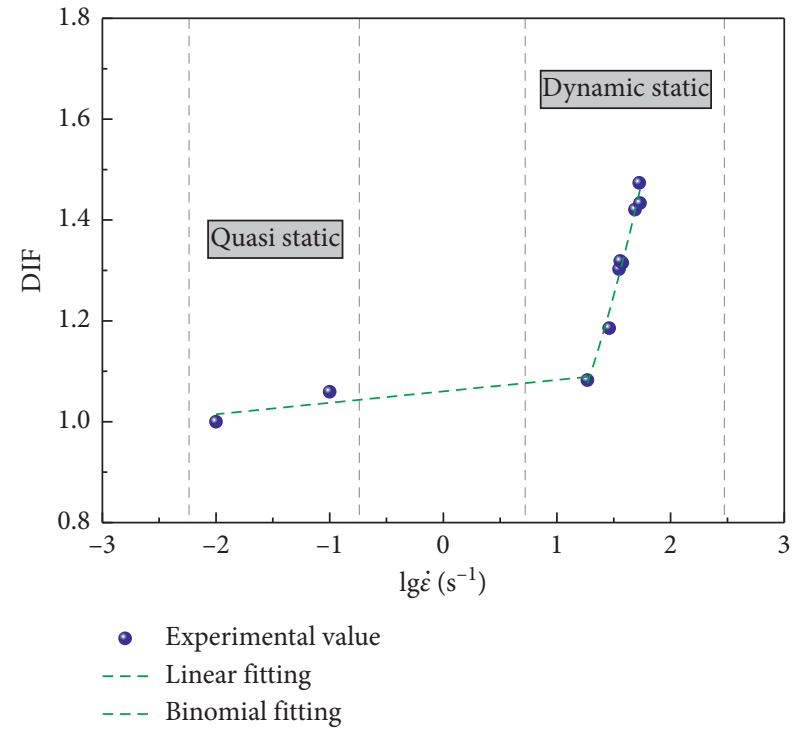

FIgURE 6: Strain rate effect on the uniaxial compressive strength.

under dynamic impact exceed $1 \%$. The strain increases nonlinearly with the increase of the strain rate, which is consistent with the considerable experimental results summarized by Bischoff and Perry [25]. To be more specific, the critical strain increases from $0.46 \%$ to $1.71 \%$, while the residual strain increases from $0.5 \%$ to $2.36 \%$.

The failure mode of rock under compression is a complex phenomenon which has not yet been properly explained with a standard mechanical model. Szwedzicki [26] divided rock failure into five modes: single tensile, single shear, multitensile, multishear, and complex fracture. Through considerable experimental studies, Ramsey and Chester [27] believe that rock compression failure can be divided into five modes: splitting failure, tensile shear failure, shear failure, dilatation failure, and bulging failure. They point out that rock failure mode transforms with the change of loading condition. That is, the failure mode of sandstone is affected by the strain rate.

Figure 8 depicts the failure modes of sandstone samples at different strain rates. Figures $8(a)-8(c)$ show that the failure mode of sandstone under quasi-static compression is mainly shear failure. At strain rate of $10^{-2} \mathrm{~s}^{-1}$, the sample presents unilateral conjugate oblique shear failure, and there are several unpenetrated fractures in the longitudinal direction, and the failure block is relatively complete. At the strain rate of $10^{-1} \mathrm{~s}^{-1}$, the sample presents $\mathrm{x}$-shaped conjugate oblique shear failure, and the sample forms failure cones on the upper and lower part. The peeling part is relatively broken. Figures $8(\mathrm{~d})-8(\mathrm{e})$ show that the failure mode of sandstone under dynamic compression is mainly split failure. Besides, the higher the failure strain rate is, the higher the failure degree is. It is also founded that, as the loading state changes from quasi-static to dynamic compression, the failure mode changes from shear failure to split failure.

3.4. Effect of Strain Rate on Energy Evolution. The process of rock deformation and failure is essentially the process of energy evolution in rock. The energy absorbed by the rock

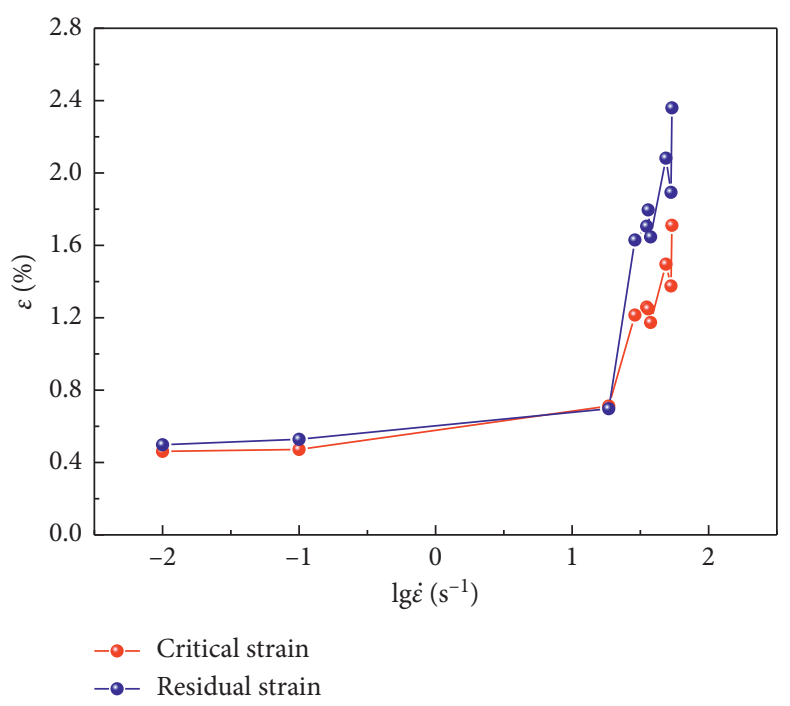

FIgURE 7: Relationship between deformation and logarithmic form of the strain rate.

during compression can be divided into recoverable elastic strain energy and unrecoverable dissipated strain energy, of which the elastic strain energy is for the elastic deformation, while the dissipated strain energy mainly consumes for the generation and development of internal microcracks $[28,29]$. Under uniaxial compression, the strain energy density of each component is calculated as follows [29]:

$$
\begin{aligned}
U & =\int_{0}^{\varepsilon} \sigma(\varepsilon) \mathrm{d} \varepsilon, \\
U_{E} & =\frac{1}{2 E} \sigma^{2}, \\
U_{D} & =\int_{0}^{\varepsilon} \sigma(\varepsilon) \mathrm{d} \varepsilon-\frac{1}{2 E} \sigma^{2},
\end{aligned}
$$

where $U, U_{E}$, and $U_{D}$ are the total strain energy density, elastic strain energy density, and dissipated strain energy density, respectively. $\sigma(\varepsilon)$ is a stress-strain function. $E$ is the unloading modulus, which is replaced by the elastic modulus when calculating.

Figure 9 presents the evolution law of each component strain energy development of sandstone at strain rates of $10^{-2} \mathrm{~s}^{-1}, 10^{-1} \mathrm{~s}^{-1}, 18.55 \mathrm{~s}^{-1}$, and $53.89 \mathrm{~s}^{-1}$ with respect to deformation. It indicated that the elastic strain energy stored in the sample increases continuously with the compression process before failure, while the dissipated strain energy changes little at a low level. When the stress reached its peak, the stored elastic strain energy is released abruptly and dissipated strain energy increases rapidly. Different from quasistatic compression, the dissipated strain energy before failure displays an obvious trend of growth in the dynamic compression process, which indicates that the stage of fracture instability is more obvious in the dynamic compression. By observing Figure 9(c), one may find that the stored elastic strain energy was not fully released after the compression process due to the incomplete failure of the sample. It is consistent with the monitored stress-strain curve. 


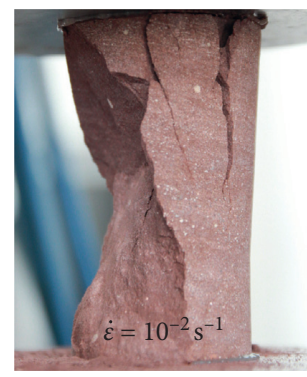

(a)

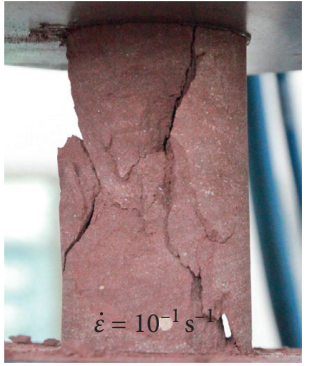

(b)

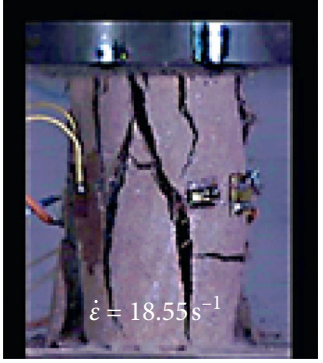

(c)

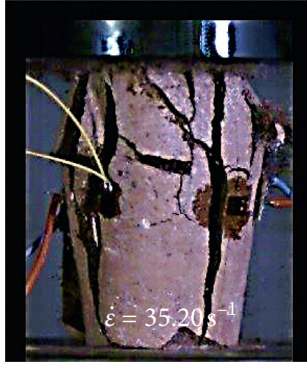

(d)

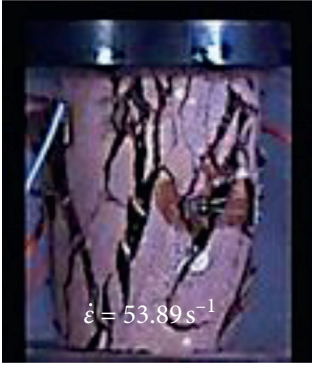

(e)

FIGURE 8: Failure mode of sandstone at different strain rates.

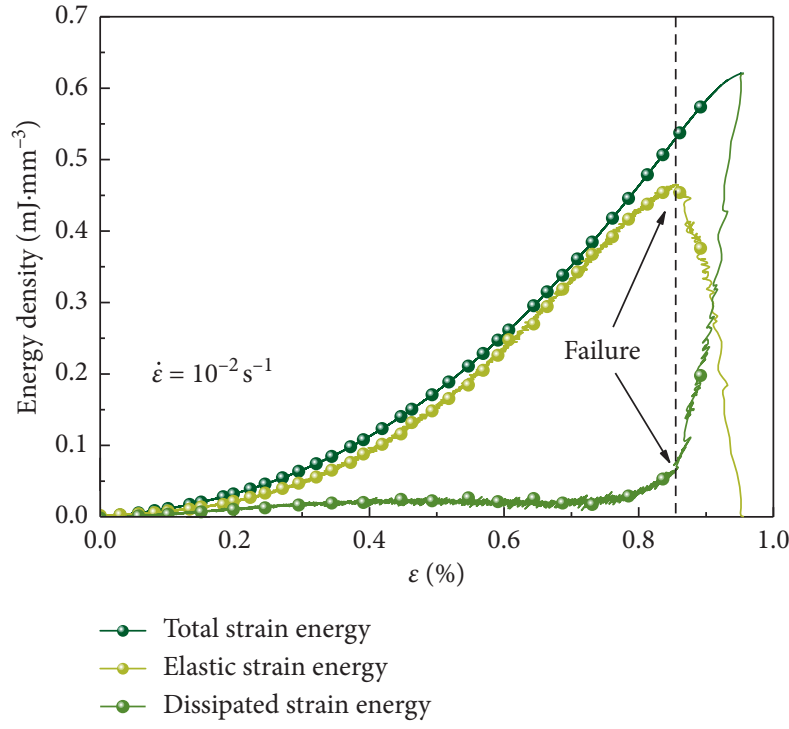

(a)

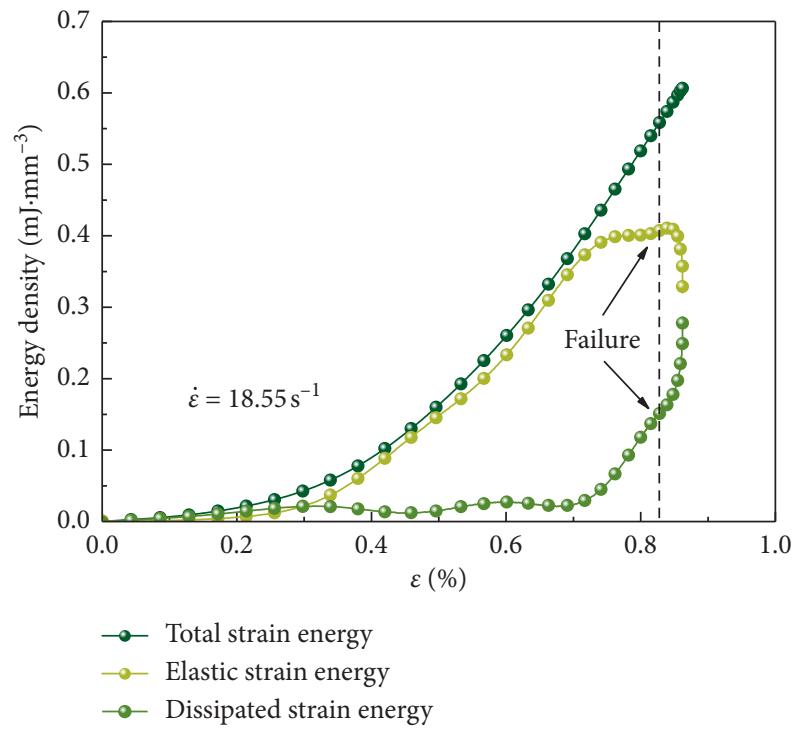

(c)

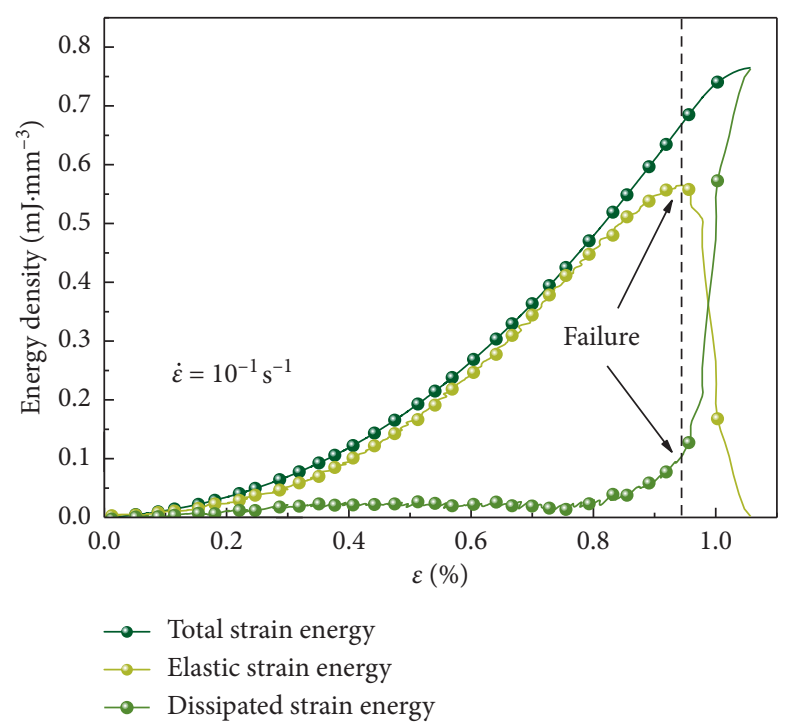

(b)

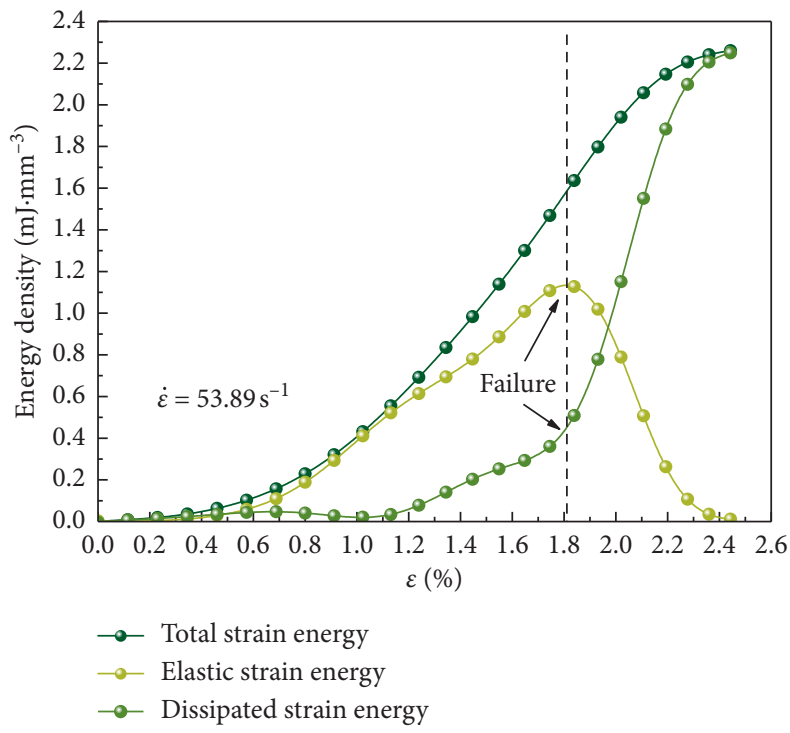

(d)

FigURE 9: Energy evolution curves of sandstone samples at different strain rates. 
Figure 10 shows the total strain energy, elastic strain energy, and dissipated strain energy of sandstone at failure time at different strain rates. It can be seen that, in the strain rate range of $10^{-2} \sim 55 \mathrm{~s}^{-1}$, the total strain energy and elastic strain energy increase significantly with the increase of the strain rate. Specifically, the total strain energy and elastic strain energy increase from $0.53 \mathrm{~mJ} /$ $\mathrm{mm}^{3}$ and $0.44 \mathrm{~mJ} / \mathrm{mm}^{3}$ to $1.69 \mathrm{~mJ} / \mathrm{mm}^{3}$ and $1.43 \mathrm{~mJ} / \mathrm{mm}^{3}$, respectively. With the increase of the loading rate, the total energy absorbed in the sample increases and the elastic unit number stored in the sample also increases abruptly, resulting in the increase of elastic strain energy [30]. The effect of the strain rate on dissipated strain energy is not obvious. The value of dissipated strain energy fluctuates in the range of $0 \sim 0.4 \mathrm{~mJ} / \mathrm{mm}^{3}$. Furthermore, the proportion of elastic strain energy and dissipated strain energy is depicted in Figure 11. It is found that the ratio of elastic strain energy and dissipated strain energy to total strain energy reflects strong discreteness at the intermediate strain rate. However, in general, with an increase in the strain rate, the proportion of elastic strain energy decreases while that of dissipated strain energy increases. Specifically, the proportion of dissipated strain energy fluctuates between $10 \%$ and $30 \%$, indicating that when the lithology and fracture conditions of the samples are similar, the strain rate has little influence on it.

3.5. Effect of Strain Rate on Brittleness. Brittleness is one of the important mechanical indexes of rock medium, whereas the evaluation standard of brittleness is not uniform. Many scholars put forward more than 20 brittleness indexes in total from the perspectives of mineral composition, strength ratio, and energy dissipation. To assess the brittleness of sandstone under impact loads, the brittleness index proposed by Baron [31] from the perspective of energy is adopted for its calculation as follows:

$$
B=\frac{W_{E}}{W},
$$

where $W$ is the total energy in the compression process and $W_{E}$ is the recoverable elastic energy. The smaller the ratio of elastic energy to total energy is, the more energy is used for destruction, which leads to the more obvious brittleness of rocks.

Figure 12 displays the change of brittleness index with the strain rate under impact loading. It can be seen that the brittleness index of sandstone decreases with the increase of strain rate within the range of $15 \sim 55 \mathrm{~s}^{-1}$, indicating that the brittleness is more obvious with the strain rate increasing. The effect of the strain rate on brittleness is closely related to energy dissipation and release during deformation and failure. With an increase in the loading strain rate, the energy consumed for fracture development increases, and the

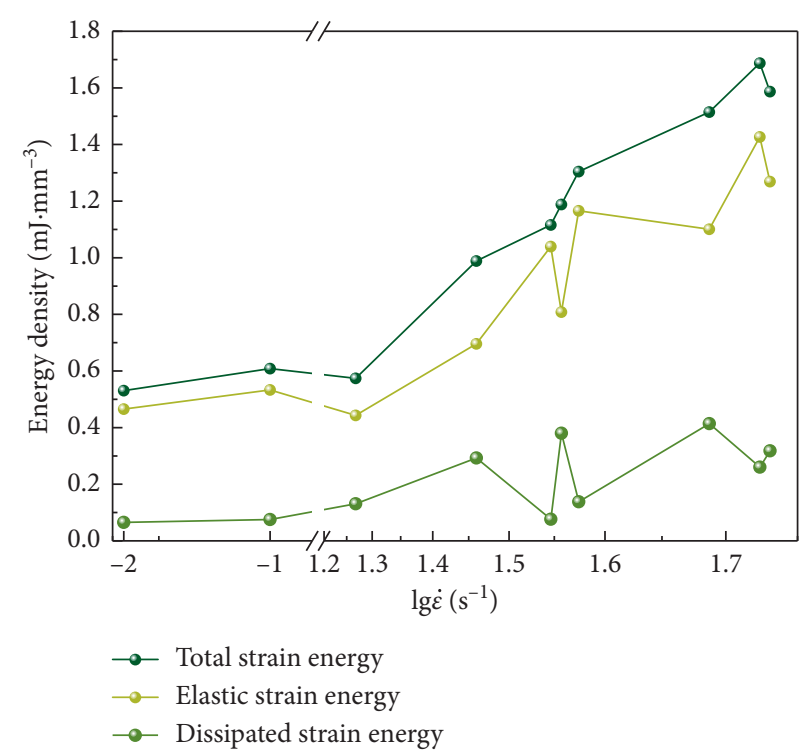

FIgURE 10: Strain rate effect on the strain energy of tested sandstone.

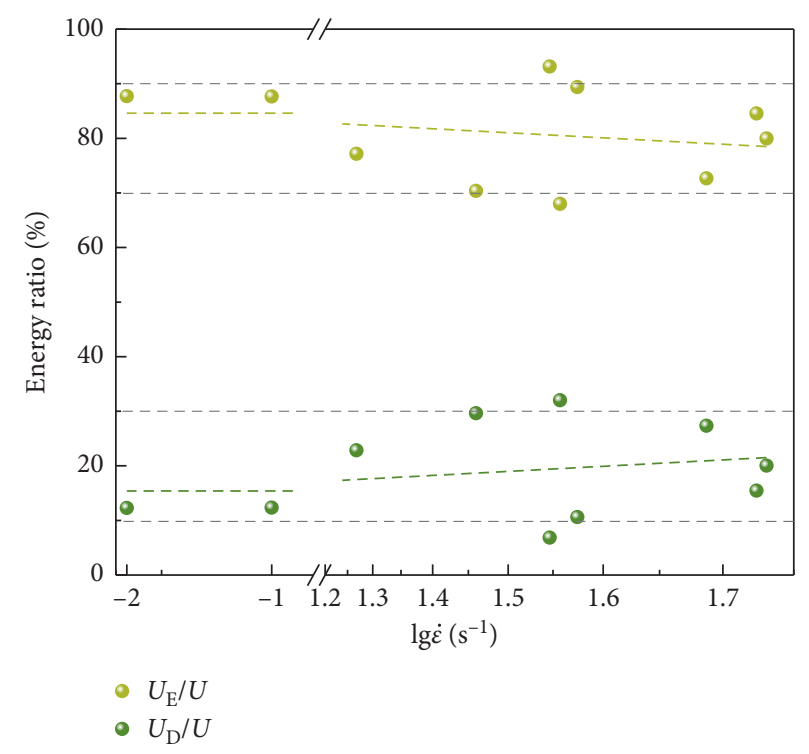

FIGURE 11: Relationship between the energy ratio and strain rate.

energy is released more dramatically during the failure, resulting in the increase of fracture degree. By fitting the experimental data, the empirical formula for the brittle index with respect to the strain rate at the intermediate strain rate is formulated:

$$
\begin{aligned}
B= & 0.73-2.99 \times 10^{-4} e^{(\dot{\varepsilon} / 10.87)}-2.99 \times 10^{-4} e^{(\dot{\varepsilon} / 12.08)} \\
& -2.99 \times 10^{-4} e^{(\dot{\varepsilon} / 13.29)} .
\end{aligned}
$$




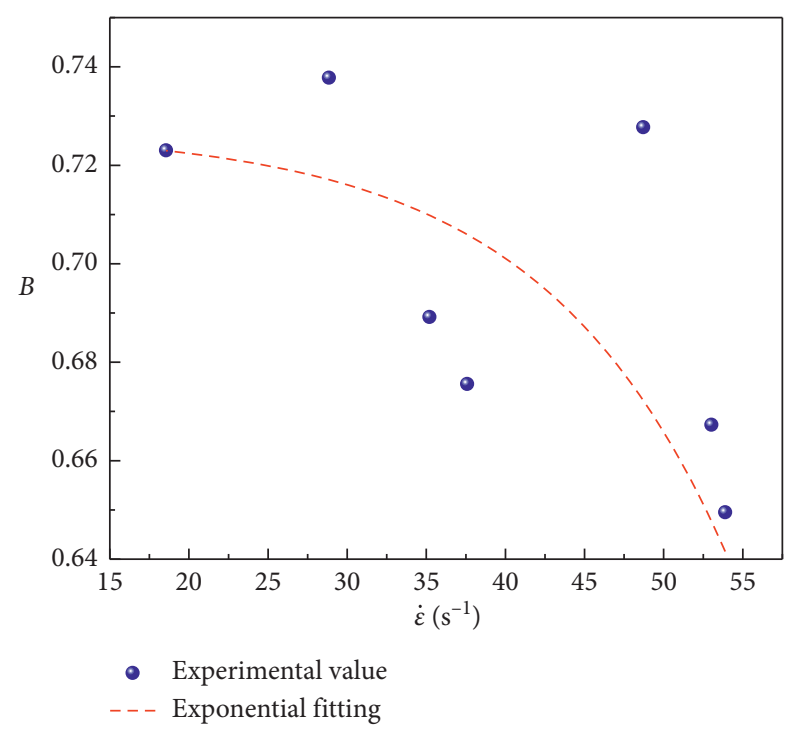

Figure 12: Strain rate effect on the brittleness index.

\section{Conclusion}

The uniaxial compression tests were carried out with MTS and drop weight impact testing machine on sandstone in the medium strain rate range of $10^{-2} \sim 55 \mathrm{~s}^{-1}$. The following main conclusions were obtained as follows.

The deformation and failure of sandstone under compression at the intermediate strain rate undergoes pore compaction stage, elastic deformation stage, instable fracture prepeak propagation stage, and postpeak descending stage. The critical strain and residual strain exhibit an increasing trend with the increase of the strain rate.

The strain rate has an evident effect on the compressive strength of sandstone. The compressive strength increases linearly with the strain rate under the quasi-static condition and increases under the dynamic condition. The failure mode of sandstone is closely related to the loading strain rate. As the loading mode changes from quasi-static to dynamic, the failure mode turns from shear failure to split failure.

The strain rate has an effect on the elastic strain energy and dissipated strain energy at failure time during energy evolution. Both elastic strain energy and dissipated strain energy increase with an increase in the strain rate, and the elastic strain energy is more sensitive to the loading strain rate than the dissipated strain energy. When the lithology and fracture conditions are similar, the strain rate has little influence on the proportion of dissipated strain energy to total strain energy.

The failure of sandstone is a typical brittle failure. The brittle characteristics of sandstone become more remarkable with the increase of the strain rate, accompanied with a higher degree of fracture.

\section{Data Availability}

All data used to support the findings of this study are available from the first author upon request.

\section{Disclosure}

Jie Shi and Zongmu Luo are first authors.

\section{Conflicts of Interest}

The authors declare that there are no conflicts of interest regarding the publication of this paper.

\section{Acknowledgments}

This work was financed and fully supported by the National Natural Science Foundation of China (Grant nos. 51774295 and 51808551), which are greatly appreciated by the authors.

\section{References}

[1] Q. B. Zhang and J. Zhao, "A review of dynamic experimental techniques and mechanical behaviour of rock materials," Rock Mechanics and Rock Engineering, vol. 47, no. 4, pp. 1411-1478, 2014.

[2] B. Huang and J. Liu, "The effect of loading rate on the behavior of samples composed of coal and rock," International Journal of Rock Mechanics and Mining Sciences, vol. 61, pp. 23-30, 2013.

[3] D. L. Grote, S. W. Park, and M. Zhou, "Dynamic behavior of concrete at high strain rates and pressures: I. experimental characterization," International Journal of Impact Engineering, vol. 25, no. 9, pp. 869-886, 2001.

[4] Q. B. Zhang and J. Zhao, "Quasi-static and dynamic fracture behaviour of rock materials: phenomena and mechanisms," International Journal of Fracture, vol. 189, no. 1, pp. 1-32, 2014.

[5] L. Ma, Z. Li, J. Liu, L. Duan, and J. Wu, "Mechanical properties of coral concrete subjected to uniaxial dynamic compression," Construction and Building Materials, vol. 199, pp. 244-255, 2019.

[6] L. Ma, J. Wu, M. Wang, L. Dong, and H. Wei, "Dynamic compressive properties of dry and saturated coral rocks at high strain rates," Engineering Geology, vol. 272, p. 105615, 2020.

[7] J. L. Shang, L. T. Shen, and J. Zhao, "Hugoniot equation of state of the Bukit Timah granite," International Journal of Rock Mechanics and Mining Sciences, vol. 37, no. 4, pp. 705-713, 2000.

[8] X. Li, F. Gong, M. Tao et al., "Failure mechanism and coupled static-dynamic loading theory in deep hard rock mining: a review," Journal of Rock Mechanics and Geotechnical Engineering, vol. 9, no. 4, pp. 767-782, 2017.

[9] F.-Q. Gong, X.-F. Si, X.-B. Li, and S.-Y. Wang, "Dynamic triaxial compression tests on sandstone at high strain rates and low confining pressures with split Hopkinson pressure bar," International Journal of Rock Mechanics and Mining Sciences, vol. 113, pp. 211-219, 2019.

[10] X. Si, F. Gong, X. Li, S. Wang, and S. Luo, "Dynamic MohrCoulomb and Hoek-Brown strength criteria of sandstone at high strain rates," International Journal of Rock Mechanics and Mining Sciences, vol. 115, pp. 48-59, 2019.

[11] K. Liu, J. Zhao, G. Wu, A. Maksimenko, A. Haque, and Q. Zhang, "Dynamic strength and failure modes of sandstone under biaxial compression," International Journal of Rock Mechanics and Mining Sciences, vol. 128, p. 104260, 2020.

[12] Z. Zhou, X. Cai, D. Ma et al., "Water saturation effects on dynamic fracture behavior of sandstone," International 
Journal of Rock Mechanics and Mining Sciences, vol. 114, pp. 46-61, 2019.

[13] J. Zhang, H. Deng, A. Taheri, B. Ke, and C. Liu, "Deterioration and strain energy development of sandstones under quasistatic and dynamic loading after freeze-thaw cycles," Cold Regions Science and Technology, vol. 160, pp. 252-264, 2019.

[14] Y. Luo, D. Qu, G. Wang, X. Li, and G. Zhang, "Degradation model of the dynamic mechanical properties and damage failure law of sandstone under freeze-thaw action," Soil $D y$ namics and Earthquake Engineering, vol. 132, p. 106094, 2020.

[15] S. Liu and J. Xu, "Effect of strain rate on the dynamic compressive mechanical behaviors of rock material subjected to high temperatures," Mechanics of Materials, vol. 82, pp. 28-38, 2015.

[16] C. Liu, Y. Wu, X.-f. Zhang et al., "Propagation of shock waves in dry and wet sandstone: experimental observations, theoretical analysis and meso-scale modeling," Defence Technology, vol. 14, no. 5, pp. 513-521, 2018.

[17] R. Ulusay, The ISRM Suggested Methods for Rock Characterization, Testing and Monitoring: 2007-2014, Springer, Berlin, Germany, 2014.

[18] M. Cai, P. K. Kaiser, F. Suorineni, and K. Su, "A study on the dynamic behavior of the Meuse/Haute-Marne argillite," Physics and Chemistry of the Earth, Parts $A / B / C$, vol. 32, no. 8-14, pp. 907-916, 2007.

[19] C. Qi, M. Wang, and Q. Qian, "Strain-rate effects on the strength and fragmentation size of rocks," International Journal of Impact Engineering, vol. 36, no. 12, pp. 1355-1364, 2009.

[20] P. Rossi and F. Toutlemonde, "Effect of loading rate on the tensile behaviour of concrete: description of the physical mechanisms," Materials and Structures, vol. 29, no. 2, pp. 116-118, 1996.

[21] M. E. Kipp, D. E. Grady, and E. P. Chen, "Strain-rate dependent fracture initiation," International Journal of Fracture, vol. 16 , no. 5, pp. 471-478, 1980.

[22] P. Rossi, "A physical phenomenon which can explain the mechanical behaviour of concrete under high strain rates," Materials and Structures, vol. 24, no. 6, pp. 422-424, 1991.

[23] D. E. Grady, "Local inertial effects in dynamic fragmentation," Journal of Applied Physics, vol. 53, no. 1, pp. 322-325, 1982.

[24] Q. Qian, C. Qi, and M. Wang, "Dynamic strength of rocks and physical nature of rock strength," Journal of Rock Mechanics and Geotechnical Engineering, vol. 1, no. 1, pp. 1-10, 2009.

[25] P. H. Bischoff and S. H. Perry, "Compressive behaviour of concrete at high strain rates," Materials and Structures, vol. 24, no. 6, pp. 425-450, 1991.

[26] T. Szwedzicki, "A hypothesis on modes of failure of rock samples tested in uniaxial compression," Rock Mechanics and Rock Engineering, vol. 40, no. 1, pp. 97-104, 2007.

[27] J. M. Ramsey and F. M. Chester, "Hybrid fracture and the transition from extension fracture to shear fracture," Nature, vol. 428, no. 6978, pp. 63-66, 2004.

[28] P. Wang, J. Xu, X. Fang, and P. Wang, "Energy dissipation and damage evolution analyses for the dynamic compression failure process of red-sandstone after freeze-thaw cycles," Engineering Geology, vol. 221, pp. 104-113, 2017.

[29] H. Xie, L. Li, R. Peng, and Y. Ju, "Energy analysis and criteria for structural failure of rocks," Journal of Rock Mechanics and Geotechnical Engineering, vol. 1, no. 1, pp. 11-20, 2009.

[30] Y. Jiang, H. LI, and Y. Zhao, "Effect of loading rate on energy accumulation and dissipation in rocks," Journal of China University of Mining \& Technology, vol. 43, no. 3, in China, 2014.

[31] L. I. Baron, Determination of Properties of Rocks, Gozgotekhizdat, Moscow, Russia, in Russion, 1962. 